\title{
Hamlet dans la culture populaire : le cas du Stick Figure Hamlet de Dan Carroll
}

\section{Pierre Kapitaniak}

\section{(2) OpenEdition \\ Journals}

\section{Édition électronique}

URL : http://journals.openedition.org/shakespeare/3662

DOI : 10.4000/shakespeare.3662

ISSN : 2271-6424

\section{Éditeur}

Société Française Shakespeare

\section{Référence électronique}

Pierre Kapitaniak, "Hamlet dans la culture populaire : le cas du Stick Figure Hamlet de Dan Carroll », Actes des congrès de la Société française Shakespeare [En ligne], 34 | 2016, mis en ligne le 10 mars 2016, consulté le 30 avril 2019. URL : http://journals.openedition.org/shakespeare/3662 ; DOI : $10.4000 /$ shakespeare.3662

Ce document a été généré automatiquement le 30 avril 2019.

(c) SFS 


\title{
Hamlet dans la culture populaire : le cas du Stick Figure Hamlet de Dan Carroll
}

\author{
Pierre Kapitaniak
}

1 Lorsqu'en 2006 parut Shakespeare and Youth Culture, Kevin J. Wetmore, qui y consacrait un chapitre à la bande dessinée et au roman graphique ${ }^{1}$, esquissait un panorama des adaptations shakespeariennes en bande dessinée où dominaient plusieurs générations de "Shakespeare Illustré », s'adressant ouvertement au jeune public, mais n'offrant que des réductions drastiques des intrigues et seulement quelques dialogues originaux. En 2006, ce constat validait de façon convaincante la tendance à ce que les auteurs appelaient dans l'introduction « la réduction, la traduction et les mentions » de Shakespeare (« Reducing, Translating, and Referencing Shakespeare »). Il est tentant d'établir un parallèle entre cette "réduction» pour la jeunesse, et la tendance réductrice qu'affiche la culture populaire lorsqu'elle s'attaque à l'icône culturelle qu'est Shakespeare. Bien que Macbeth soit sa pièce la plus adaptée de nos jours, tant au cinéma qu'au théâtre, c'est pourtant Hamlet qui représente la quintessence de l'œuvre de cette icône de la littérature mondiale. Dans la culture populaire, la pièce se retrouve réduite à des formules ou slogans, des expressions ou citations célèbres qui, par métonymie, se substituent à la pièce. Deux d'entre elles semblent s'être imposées sans conteste: le premier vers du monologue de Hamlet ( « To be or not to be », III.i.64), le seul à fonctionner dans la langue originale quelle que soit la langue de la culture populaire et, quoique moins référencé, le commentaire de Marcellus, « Something is rotten in the state of Denmark» (I.iv.100), qui convoque lui aussi immédiatement la tragédie. En plus de ces réductions textuelles, la fameuse tragédie est également et fréquemment réduite à une représentation graphique, qui emprunte à la scène des fossoyeurs l'image du prince contemplant le crâne de Yorick (V.i.190-191). Avec le temps, les deux types de réduction - l'icône et le slogan - ont fusionné en un agrégat synthétique immédiatement reconnaissable et identifiable, " To be or not to be » venant à remplacer « Alas poor Yorick».

2 Lorsque l'on souhaite évoquer Hamlet, ce sont ces trois éléments qu'on retrouve immanquablement, et ils sont souvent associés. Pour ne citer qu'un exemple, on les 
retrouve tous trois dans la façon dont Albert Uderzo et René Goscinny évoquent Hamlet dans le $22^{\mathrm{e}}$ volume des aventures d'Astérix et Obélix (La Grande traversée, 1975). Dans les deux allusions à Hamlet situées sur deux planches peu éloignées l'une de l'autre, les auteurs combinent ces trois réductions qui accompagnent inévitablement la visite de nos héros au Danemark ${ }^{3}$. On pourrait consacrer d'épais ouvrages aux exemples de telles iconisations de Hamlet, dans la bande dessinée, au cinéma ou dans d'autres médias, tant ce type d'appropriation extrême du capital culturel représenté par Shakespeare est fréquent dans une culture de la fragmentation exacerbée par la télévision et internet. Cette appropriation a été souvent perçue de façon négative, décrite en termes moralisateurs comme un appauvrissement ou une trahison. La bande dessinée a tout autant souffert de tels jugements, en partie sans doute parce que ce mode d'expression artistique est censé s'adresser traditionnellement à un public jeune. Or, dans les pages qui suivent, je voudrais me pencher sur le cas de Stick Figure Hamlet de Dan Carroll ${ }^{4}$ que l'on pourrait situer aux antipodes de la réduction iconique ou de la simplification pédagogique, témoignant peutêtre d'une évolution de la bande dessinée.

\section{Shakespeare et la bande dessinée : des classiques illustrés à Stick Figure Hamlet}

3 Tout au long du $\mathrm{XX}^{\mathrm{e}}$ siècle, les rapports entre la bande dessinée et Hamlet semblaient surtout didactiques. Les jeunes lecteurs de l'après-guerre pouvaient découvrir la fameuse tragédie dans des adaptations illustrées, qui combinaient des passages du texte shakespearien avec des résumés et paraphrases pour offrir une publication d'une cinquantaine de pages. Dans les années 1950, deux éditeurs publièrent des versions concurrentes : une première en 1950 dans la série Famous Authors Illustrated, illustrée par Henry Kiefer ${ }^{5}$ et une seconde illustrée par Alex A. Blum en 1952 dans la série Classics Illustrated ${ }^{6}$. Cette dernière série eut d'ailleurs une seconde vie dans les années 1990 et la nouvelle version de Hamlet fut illustrée par Tom Mandrake en 1991'. À la suite de cette seconde série, d'autres éditeurs se sont engouffrés dans la brèche comme le Saddleback's Illustrated Classics (2003), Picture This! Shakespeare (2006), ou encore la série Graffex (2009), auxquels on peut ajouter des projets individuels comme le Hamlet illustré par Leonid Gore et réécrit par Bruce Coville (2004) $)$. Entre-temps, c'est dans l'Europe des années soixantedix que l'on rencontre davantage d'originalité. En 1975, Gotlib fait paraitre une adaptation très brève de Hamlet, plus sur le mode de la plaisanterie et de l'allusion que d'une véritable adaptation de l'histoire, puisque l'ensemble se réduit à neuf pages. Et, l'année suivante en Italie, Gianni De Luca offre une adaptation graphiquement très innovante ${ }^{10}$, mais dont le texte a dû subir une forte censure du Vatican.

Dan Carroll n'est donc pas le premier à adapter la tragédie de Hamlet au média de la bande dessinée. Cela dit, lorsqu'il a commencé à travailler sur Stick Figure Hamlet en 2005, il n'en existait aux États-Unis que des versions très réduites et souvent très pédagogiques, accompagnées d'un dossier sur l'auteur et son contexte. En 2005, son idée d'illustrer l'intégralité de Hamlet apparaît donc comme un projet à la fois ambitieux et original ${ }^{11}$. Avant même que le Stick Figure Hamlet ne soit terminé (Dan Carroll mettra 4 ans à terminer son projet), d'autres adaptations ambitieuses relevant notamment du genre manga voient le jour : une adaptation futuriste par la britannique Emma Vieceli (2007) et une autre tournée vers le genre médiéval-fantastique par Tintin Pantoja et Adam Sexton $\left(2008^{12}\right)$. Cette fois le format est plus étendu, avoisinant deux centaines de pages, et leurs 
auteurs mêlent texte original, réécritures modernisées et descriptions ajoutées. C'est probablement en raison de ce développement que l'adaptation graphique de Hamlet dans la série No Fear Shakespeare qui paraît en 2008 adopte un format également étendu de deux centaines de pages $^{13}$. On remarquera d'ailleurs que l'évolution des couvertures de ces adaptations reflète mes remarques préliminaires sur la réduction de Hamlet au crâne de Yorick. À partir du tournant du siècle, les couvertures déclinent presque invariablement le motif graphique du prince tenant le crâne, alors que les premières préféraient l'apparition du fantôme (Illustrations 1 à 3). Et notre auteur n'échappe pas à cette tendance.

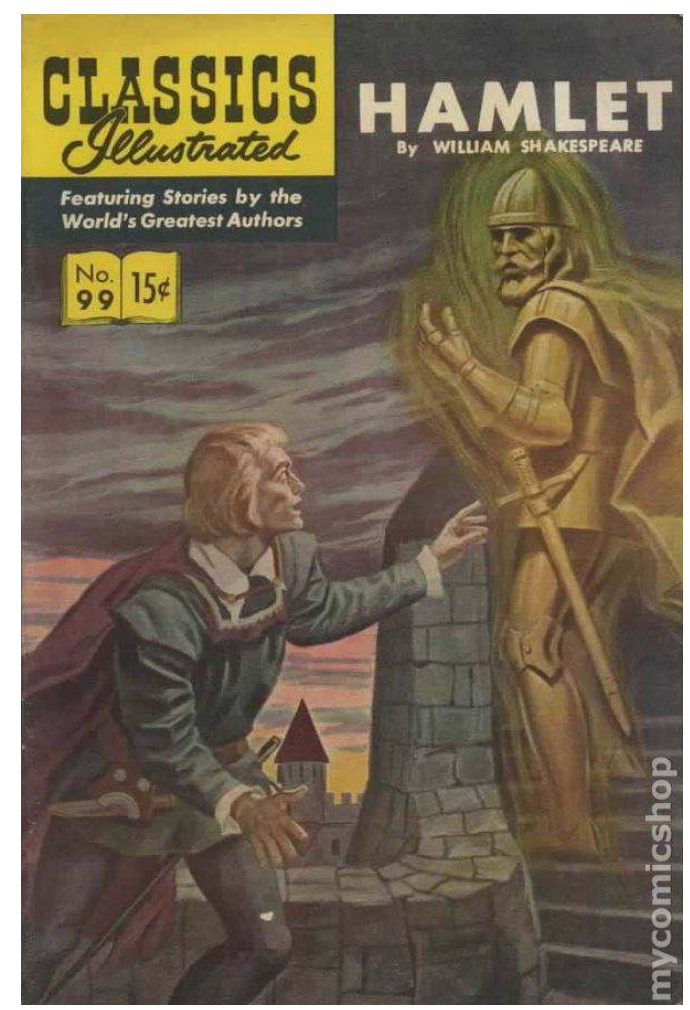

Illustration 1 : Classics Illustrated: Hamlet, illustré par Alex A. Blum, Gilberton, 1952.

Couverture

URL : http://www.amazon.fr/Classics-Illustrated-39-William-Shakespeare/dp/190681466X/ ref=sr_1_4?ie=UTF8\&qid=1455038339\&sr=8-4\&keywords=Classics+Illustrated\%3A+Hamlet+Alex+A. + Blum 


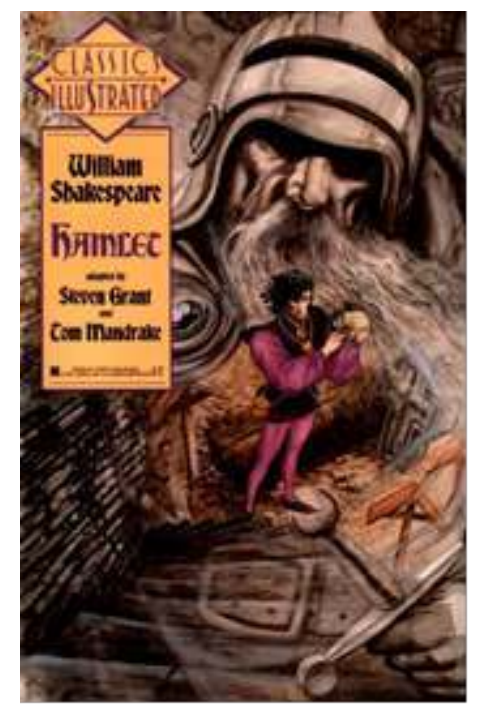

Illustration 2 : Classics Illustrated: Hamlet, illustré par Tom Mandrake, First Classics Inc., 1991.

Couverture.

URL : http://www.amazon.com/Classics-Illustrated-First-comic-book/dp/B01AV1MLGS/

ref=sr_1_cc_1 ? s=aps\&ie=UTF8\&qid=1455038840\&sr=1-1-catcorr\&keywords=hamlet + mandrake

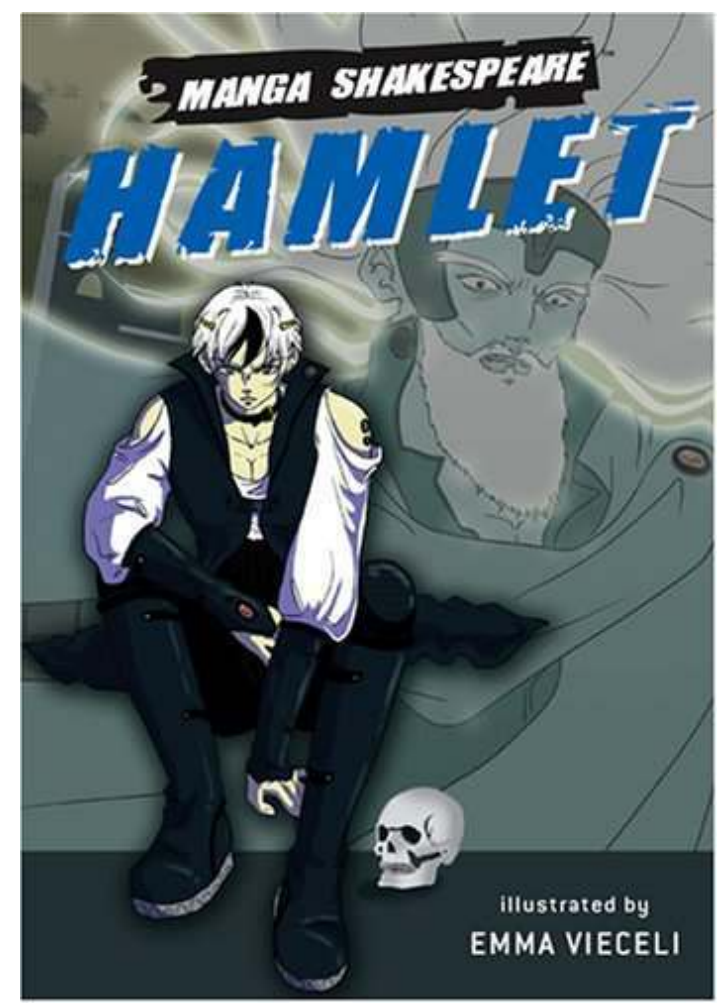

Illustration 3 : Hamlet, illustré par Emma Vieceli, Amulet Books, 2007.

Couverture.

URL : http://www.amazon.com/Manga-Shakespeare-Hamlet-William/dp/0810993244

5 Pour mener à bien son projet, Dan Carroll opte pour un type très particulier de bande dessinée, qui s'est développé avec l'avènement d'internet. Il commence la publication de 
Stick Figure Hamlet dans un blog sous forme de webcomic avec une page ajoutée chaque lundi, mercredi et vendredi, entre 2005 et 2008. Le terme de webcomic apparaît vers 1995, mais correspond à un média qui a fait son apparition au milieu des années 1980. L'avantage de ce mode d'expression est qu'il permet de publier périodiquement les pages de l'histoire sur un blog, comme le faisaient les auteurs des générations précédentes dans la presse hebdomadaire ou mensuelle, mais sans contrat ni investissement, en conservant une totale liberté d'expression ainsi que le contrôle du flux, de la forme et du contenu. Les premiers webcomics ont exploité prioritairement la veine parodique, comme Witches and Stitches d'Eric Milikin qui offrait une variation décalée sur Le Magicien d'Oz.

Comme son titre l'indique, en plus du format de webcomic, Carroll choisit une approche minimaliste qui réduit ses personnages à des bonshommes-allumettes ou «stick figures ", procédé graphique développé surtout par les spécialistes de la communication et de la signalétique, mais très vite adopté par les auteurs de webcomics, en raison du caractère facilement accessible de cette charte graphique très simple, ce qui la rend également adaptable au dessin assisté par ordinateur. Le premier webcomic en bonhomme-allumette - NetBoy de Stafford Huyler - démarra à l'été 1994 sous forme de blog, et continue encore, le dernier post datant du 10 juin $2014^{14}$.

\section{Adaptation et motivation}

7 Si d'autres auteurs de bande dessinée ont déjà illustré le texte intégral d'autres pièces de Shakespeare, Dan Carroll est le premier à le faire pour Hamlet. Mais contrairement aux divers exemples que j'ai évoqués plus haut, faut-il encore parler d'adaptation? Pour reprendre la définition de Linda Hutcheon dans A Theory of Adaptation, l'adaptation correspond à trois aspects :

An acknowledged transposition of a recognizable other work or works

A creative and an interpretive act of appropriation/salvaging

An extended intertextual engagement with the adapted work ${ }^{15}$.

Un peu plus loin, Linda Hutcheon étend ce concept déjà très inclusif à tout passage d'un mode narratif (qu'elle appelle "telling») à un mode scopique ("showing») : «In a very real sense, every live staging of a printed play could theoretically be considered an adaptation in its performance ${ }^{16}$.» En soi, ce postulat se complique pour le théatre élisabéthain. Même si un texte manuscrit précède nécessairement toute représentation, on ne peut en dire autant du texte imprimé et publié, puisque pour les pièces de l'époque ce dernier ne précédait jamais les représentations. Mais c'est un autre débat.

Dans le cas qui m'intéresse ici, Dan Carroll choisit d'illustrer le texte modernisé de la version longue du second in-quarto de 1604/1605, et à quelques variantes textuelles près le texte se rapproche de celui qui est mis en ligne par la Folger Library. Dans la mesure où Carroll n'opère que des modifications très minimes sur le texte fourni (quelques rares mots de liaison qui sont supprimés), Stick Figure Hamlet est bien plus proche d'une mise en scène que d'une adaptation, ou du moins d'une adaptation cinématographique qui conserverait le texte "intégral », comme a pu le faire par exemple Kenneth Branagh pour son Hamlet de $1996^{17}$. Comme au cinéma et au théâtre, les didascalies sont traduites visuellement. Comme au cinéma, la bande dessinée propose au lecteur une vision cadrée, dirigée, limitant la liberté que le spectateur de théâtre conserve pour se focaliser sur tel ou tel point dans l'espace offert par la mise en scène. À la différence du cinéma, cependant, qui conserve la dimension cinétique du spectacle vivant, le média de la bande 
dessinée contraint l'auteur à figer l'image sur une planche et donc à sélectionner un nombre limité d'images qui distillent en résumé l'action fluide du film ou de la pièce. Ainsi, la bande dessinée peut être vue comme existant à mi-chemin entre le texte théâtral imprimé et sa mise en scène vivante.

Dans son examen des différents modes d'adaptation, Hutcheon met l'accent sur les motivations qui conduisent l'adaptateur à s'emparer d'une œuvre. En général, la première motivation est de profiter d'une manne financière, le succès de l'œuvre source garantissant par capillarité une certaine notoriété de l'adaptation. Or, dans le cas de Dan Carroll, cette motivation semble secondaire, puisque pendant les quatre années de création le projet ne générait aucune recette. À titre de comparaison on peut évoquer le récent projet d'un autre auteur de webcomics, Ryan North, dont la gestion a été à l'opposé de celle de Carroll. Renouant avec un genre qui fit son apparition dans les années 1980, North a lancé en 2012 le projet d'adapter Hamlet au format de "Choose Your Own Adventure " (en français les "livres dont vous êtes le héros »), en choisissant la plateforme Kickstarter qui permet de faire appel aux dons des internautes, en échange desquels le travail de mise en ligne progresse. En très peu de temps, ce Hamlet dont le lecteur est le héros devint l'une des aventures les plus lucratives de la plate-forme en totalisant plus de 500000 dollars de promesses de dons ${ }^{18}$.

L'argent n'était donc pas la motivation principale de Carroll et, de plus, Hamlet était son premier projet de webcomic (il en a publié deux autres depuis). Hutcheon recense d'autres types de motivation, parmi lesquels l'« ascension sociale » (« adaptation upwardly mobile ») et l'« élan pédagogique» ("pedagogical impulse») ${ }^{19}$. Pour la première, elle cite l'exemple des débuts du cinéma, qui en adaptant des classiques de la littérature cherchait à se forger une légitimité culturelle. Pour le second, surtout lorsqu'il s'agit de réduction, la motivation est de rendre accessible une œuvre au plus grand nombre. On retrouve ces deux types de motivation dans le contraste entre les deux pages de titre qui ouvrent le webcomic sur le site. L'hyperbole de la première (« The greatest work in human history... now with pictures »), digne des blockbusters hollywoodiens, reflète clairement la volonté de rendre ce chef-d'œuvre plus accessible à l'aide d'images. L'euphémisme et l'oralité de la langue dans la seconde (" As adapted by Dan Carroll from this play he read one time ») suggèrent le désir de reléguer Shakespeare au second plan, là encore de manière ironique, un peu comme Gus Van Sant l'avait fait pour son adaptation libre des Henry IV intitulée My own Private Idaho, où le nom de Shakespeare n'apparait qu'au générique de fin, discrètement glissé au milieu de tant d'autres collaborateurs : «Additional dialogue by William Shakespeare ». En effet, reprenant Marjorie Garber, Hutcheon rappelle que Shakespeare est la cible rêvée pour ce type d'adaptation :

Adaptations of Shakespeare, in particular, may be intended as tributes or as a way to supplant canonical cultural authority. As Marjorie Garber has remarked, Shakespeare is for many adapters "a monument to be toppled"

Si l'on peut se fier à l'interview de Carroll sur la genèse de Stick Figure Hamlet, c'est le capital culturel incarné par Shakespeare qui en a été la motivation première :

A few years ago, as a brainstorming activity, I was having friends throw one-liner titles at me, so I could draw them a funny picture. One of my friends suggested The Winter of Our Discontent, and I drew him a goofy stick figure for Richard III, standing in the snow. In order to make him a hunchback, I gave him a weird oval body, and I liked the look of it. It was a dopey little drawing, but I just liked how it looked... and decided that Stick Figure Shakespeare would be a hilariously insane project, just devoting a 300-page comic to one single joke, over and over again. I decided on Hamlet very quickly - it wouldn't be as funny to do an irreverent, cartoony version 
of a comedy. I wanted to go with the most overwrought, emotional tragedy I could find, and that was Hamlet ${ }^{21}$.

Carroll met en avant l'essence ludique de son adaptation depuis la description romancée de sa genèse, avec la multiplication d'épithètes comme " funny », " goofy ", « weird », et « dopey », jusqu'au projet lui-même, décrit comme " hilariously insane », " cartoony » et «irreverent». C'est seulement par la suite que la préoccupation pédagogique ou éducative s'est comme greffée sur le projet de façon interactive :

The biggest thing that ended up influencing it was that around halfway through the

first act, I started getting e-mails from teachers telling me that they loved it, and

they wanted to use it to teach their students ${ }^{22}$.

\section{Stratégies d'adaptation}

11 C'est donc en fonction de ces motivations qu'il faut analyser les choix et les stratégies mis en place dans Stick Figure Hamlet comme autant de gestes de mise en scène. Certains choix sont dictés par le média adopté et les conventions graphiques retenues; d'autres peuvent s'expliquer par le désir didactique d'expliciter un texte difficile; d'autres, enfin, permettent de déduire une lecture et interprétation personnelle de la pièce. Or, comme nous allons le voir, ces trois catégories se recoupent la plupart du temps, fruit de négociations entre contraintes techniques et interprétation personnelle.

Contrairement à la plupart des adaptations graphiques de Hamlet, Dan Carroll choisit d'effacer le plus possible tout marqueur temporel qui dénoterait une époque particulière. Même si ce choix n'est pas inévitablement dicté par la convention graphique du «bonhomme-allumette ", il reste en parfaite adéquation avec la nature minimaliste du procédé, fournissant à des personnages réduits à quelques traits distinctifs, un cadre tout aussi dépouillé. De fait, l'univers de ce Hamlet essaie d'introduire le moins d'anachronismes possible. C'est ce que confie l'auteur dans une interview :

It was a decision I made early on - whenever possible, avoid anachronisms. I didn't want to flagrantly insert anything that would break the time period... thought I still cheat about that, Rosencrantz and Guildenstern are blatantly done as Bert and Ernie, the ghost in the first scene is a Pacman ghost ${ }^{23}$.

13 Le spectre a effectivement la forme des fantômes dans le fameux jeu d'arcade, à laquelle Carroll ajoute une couronne ainsi que des bras et des jambes. Le traitement de Rosencrantz et Guildenstern est plus complexe que l'inspiration avouée de Sesame Street, car la première fois qu'ils apparaissent à l'acte II, scène i, leur mise en situation renvoie au début du film que Tom Stoppard a adapté de sa propre pièce, Rosencrantz and Guildenstern are Dead (1990), et qui montre les deux protagonistes aux prises avec l'impossible série de probabilités qui survient dans leur partie de "pile ou face » (Illustration 4). À ces deux concessions, il faut ajouter deux clins d'œil tout aussi flagrants et empruntés surtout à la culture cinématographique : l'un à l'univers de Disney, lors de la scène du verger contée par le spectre à l'acte $\mathrm{I}$, scène $\mathrm{v}$, qui utilise un cliché de Bambi en guise de décor idyllique (Illustration 5); l'autre à l'affiche du Hamlet de Franco Zeffirelli (1990), qui reproduit le même agencement des personnages autour de Hamlet à l'exception du fantôme (Illustrations 6 et 7). 


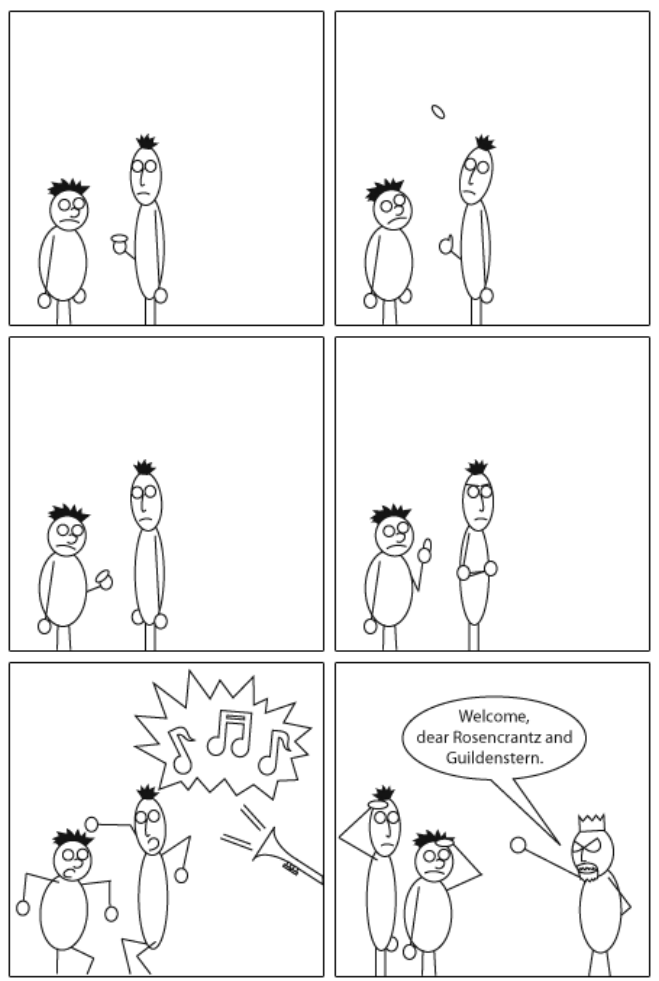

Illustration 4 : Stick Figure Hamlet, Acte II, scène i.

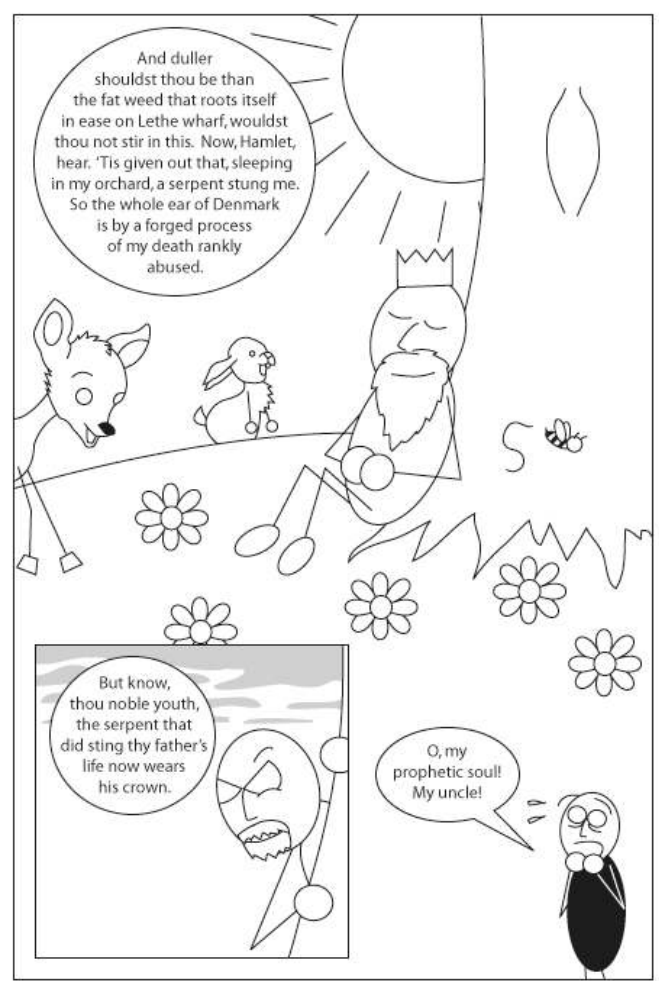


Illustration 5 : Stick Figure Hamlet, Acte I, scène v.

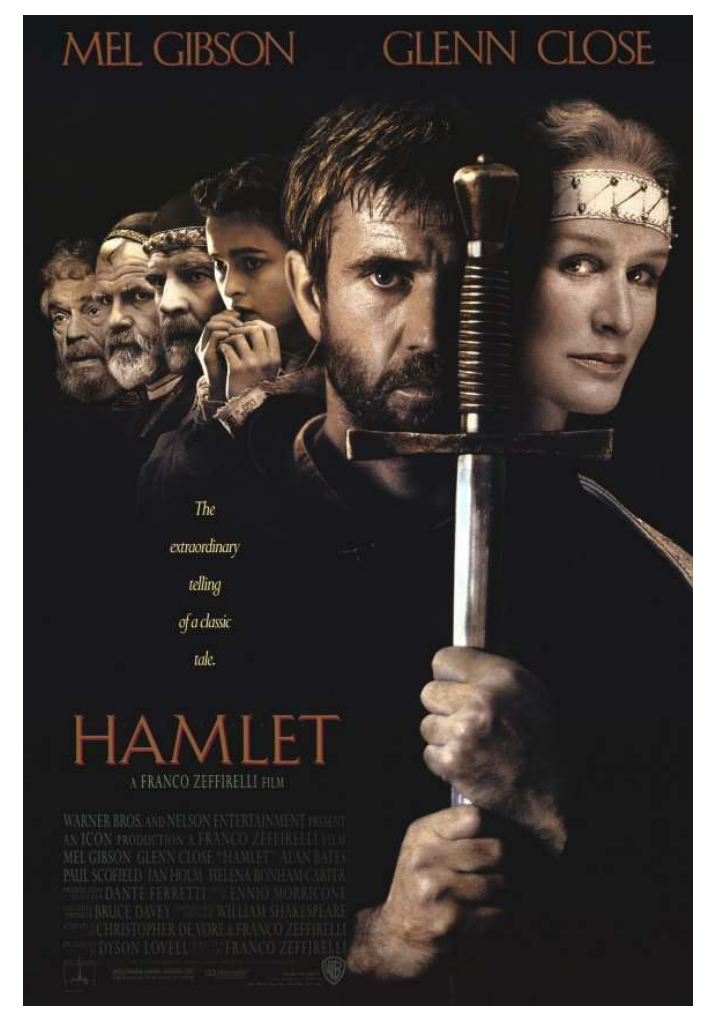

Illustration 6 : Franco Zeffirelli, Hamlet, 1990.

Affiche.

Production : Nelson Entertainment, Icon Productions, Carolco Pictures. Distribution : Time Warner, StudioCanal / Universal Pictures. 


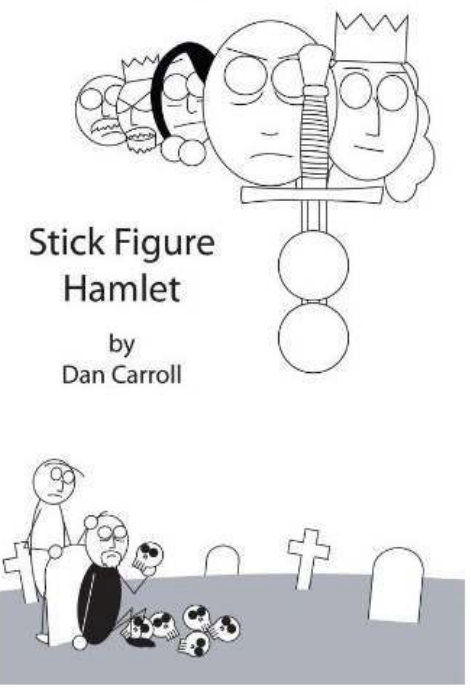

Illustration 7 : Stick Figure Hamlet, page de titre.

Parmi les choix qui découlent plus ouvertement des conventions graphiques retenues, le plus important est de loin celui de la caractérisation des personnages. L'adaptation minimaliste en bonhomme-allumette oblige Carroll à trouver dans cette économie de moyens des astuces pour différencier les protagonistes de façon très claire. La simplification graphique de la silhouette laisse relativement peu de marge de manœuvre. Carroll joue sur la rondeur du tronc ou de la tête, s'interdit les vêtements et limite les accessoires "intégrés » à un couvre-chef (couronne pour le couple royal ou les monarques défunts) et des cheveux dont la présence devient, avec la poitrine, le trait caractéristique de la féminité (la blonde Gertrude et la brune Ophélie) ${ }^{24}$. Pour différencier les personnages masculins, il adopte un tronc entièrement noir pour Hamlet, et les autres hommes se distinguent par leur système pileux : moustache et barbichette pour Claudius, barbe plus fournie pour Hamlet père et une moustache seule pour Polonius. Les liens de sang semblent être suggérés par la forme de la tête : ovale pour Claudius, Gertrude et Hamlet, ronde pour Polonius, Laërte, et Horatio, mais pas Ophélie. Un autre trait distinctif oppose deux groupes de personnages. La plupart ont les yeux ronds, tandis que d'autres ont les yeux en amande. En fait, en raison de la caractérisation des démons évoqués par les flammes infernales, cette division semble opposer des personnages "bons" ou «neutres » aux « méchants », parmi lesquels il faut alors compter Claudius, bien sûr, mais également Laërte, Hamlet père, et Fortinbras père et fils. Cela induit une lecture de la pièce à première vue assez manichéenne car on ne peut s'empêcher d'être influencé par les dessins qui enferment ces personnages dans des rôles types. Mais en même temps, une telle lecture ouvre des possibilités d'interprétation en décalage avec le texte de la pièce. Même le spectre, qui pourtant est représenté en fantôme de Pacman, rendant impossible la ressemblance avec ce qu'il était de son vivant, participe de cette logique manichéenne puisque ses yeux sont ronds (y compris quand le fantôme est représenté comme démon 
potentiel) contrairement à ceux de Hamlet père de son vivant. La forme de la tête d'Ophélie et celle des yeux de Hamlet père permettent d'envisager pour la caractérisation des personnages une logique équivalente à une focalisation externe dont le foyer serait Hamlet: la tête ovale d'Ophélie dénoterait alors sa proximité affective avec le prince, tandis que les yeux « méchants » du père joueraient sur le complexe d'ÆEdipe.

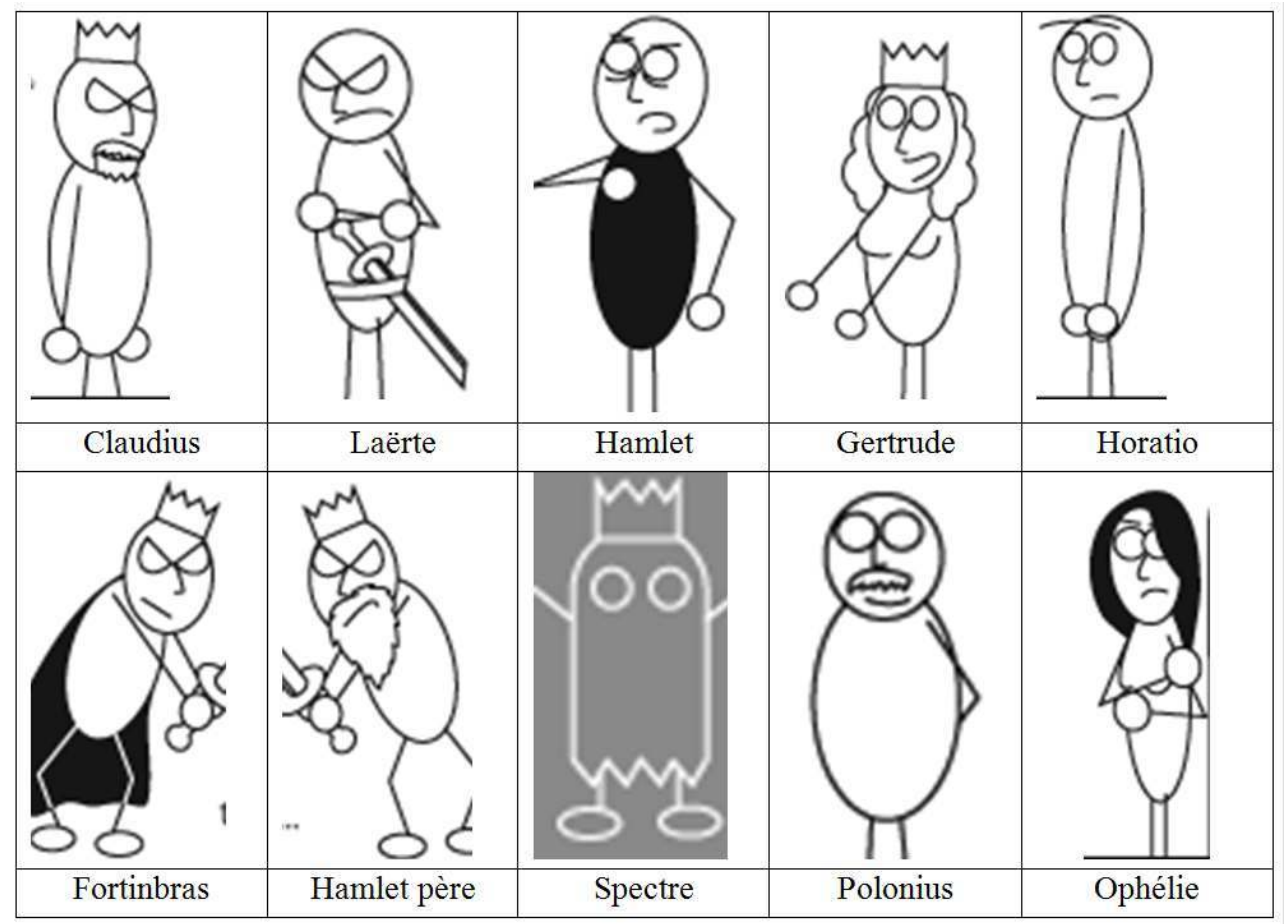

Illustration 8 : personnages de Stick Figure Hamlet.

Une autre conséquence de la convention du bonhomme-allumette est l'absence de signes visibles du manteau de la folie (« antic disposition »), puisque Hamlet n'a ni vêtements ni cheveux. Hamlet est le même à l'acte I qu'à l'acte II. En revanche, il est le seul à évoluer au cours de l'histoire. Parfaitement glabre jusqu'à son départ pour l'Angleterre, il apparaît avec une barbe de trois jours lorsqu'il se rend au bateau à l'acte IV, scène iv, et après son périple en mer il revient, à l'acte $V$, scène $i$, avec un bouc qui n'est pas sans rappeler la barbe plus fournie de son père défunt. Cette évolution unique fait ressortir Hamlet non seulement en tant que centre de la pièce mais, par rapport aux autres, fait de lui un personnage " plus en chair ", ayant une "vie » à lui. À ce propos, il est intéressant de voir que les comédiens qui viennent à Elseneur sont les seuls autres personnages à avoir un tronc noir, ce qui suggère que Carroll a souhaité conférer à Hamlet une dimension métathéâtrale, sans doute inspirée par la lecture de Stoppard. L'importance de cette dimension est reflétée davantage encore par la représentation de la folie sous forme de marionnette animée par Hamlet lorsqu'il fait ses excuses à Laërte avant le duel de l'acte $\mathrm{V}$, scène ii, ainsi que par le tableau final qui referme la bande dessinée comme une pièce de théâtre. 


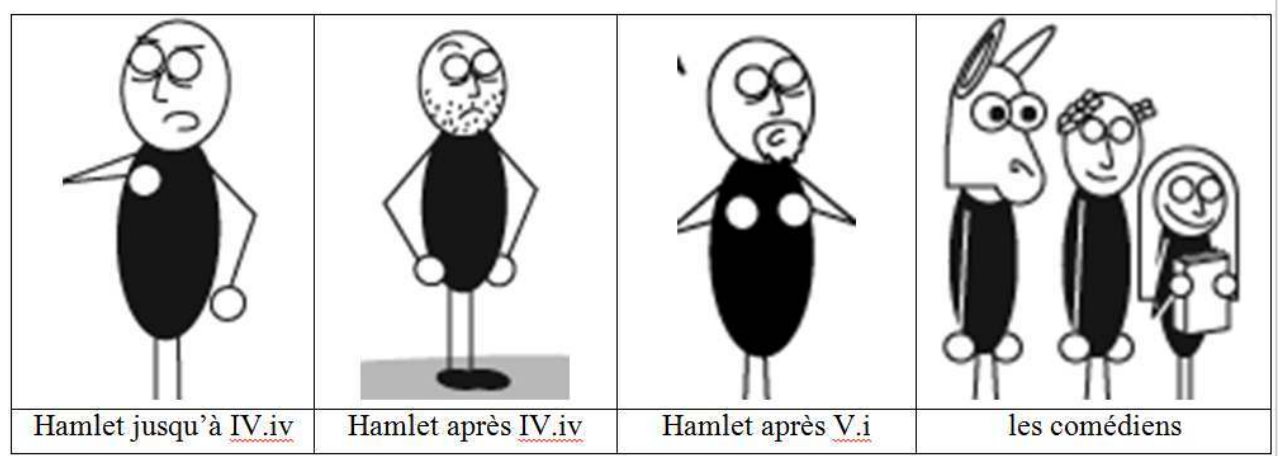

Illustration 9 : évolution de Hamlet et les comédiens de Stick Figure Hamlet.

Parmi d'autres choix qui peuvent se justifier par la nature du média dans lequel s'exprime l'adaptateur, on relève une tendance générale à doubler les hypotyposes et autres passages narratifs qui s'y prêtent par des visualisations, tendance sans doute héritée des codes cinématographiques. Les plus mémorables d'entre eux sont la description des dangers d'un spectre maléfique par les amis de Hamlet à l'acte I, scène iv, et la mort d'Ophélie à l'acte IV, scène vii.

Ce qui frappe le plus dans Stick Figure Hamlet, c'est la mise en page du texte qui abandonne délibérément toute trace de versification, y compris dans l'entretien avec les comédiens ou la pièce dans la pièce, où le pentamètre iambique rimé est délibérément déconstruit et réarrangé en phrases. On peut supposer derrière cette décision une volonté de rendre le texte moins "décourageant » pour un jeune public qui pourrait rechigner à lire de la poésie. Mais c'est surtout par l'humour, par une forme de contrepoint comique graphique, que Carroll entend exorciser toute appréhension devant la difficulté du texte. Le jeu de mots visuel ou la plaisanterie jouent effectivement un rôle central et constituent même un point de convergence de ses diverses motivations. Nous avons vu que son projet était parti d'une plaisanterie, et que Carroll l'envisageait comme «follement hilarant ». Par la suite, en avançant dans l'illustration du premier acte, il s'est rendu compte du fort potentiel comique que renferme la tragédie :

Very little work was needed for me to make it funny, and I started looking at the play with an eye towards what's already funny in here, that I can bring out ${ }^{25}$.

Dès lors, outre la mise en images de l'intrigue, le travail de Carroll a consisté soit à ajouter des effets comiques soit à souligner ceux qui existaient déjà.

L'essence ludique du projet de Carroll est effectivement servie par deux procédés assez proches : l'ajout et l'emphase graphiques. Puisque l'adaptateur a décidé de rester fidèle au texte original, l'illustration est le seul espace de liberté dont il dispose pour agir sur sa source. L'un des objectifs de ces procédés est d'expliciter le contenu du texte. C'est clairement le cas de l'élaboration d'un gag autour du passage sur le "hobby horse " (III.ii.143) où Hamlet bouscule Ophélie et la fait tomber de sa chaise. Quelques vers auparavant dans la même scène, c'est l'expression "Vulcan's stithy » (III.ii.89), dont le sens est sans doute peu transparent pour un jeune lecteur du XXI siècle, qui reçoit l'illustration d'un Hamlet enragé poursuivant Claudius une hache à la main. Ou encore l'illustration d'un Hamlet lubrique pour expliciter le sens des « unholy suits» (I.iii.138). L'exemple le plus réussi à mes yeux est l'explicitation de l'expression «edified by the margent» (V.ii.168) pour laquelle Carroll ajoute une note marginale en abyme (Illustration 10). 


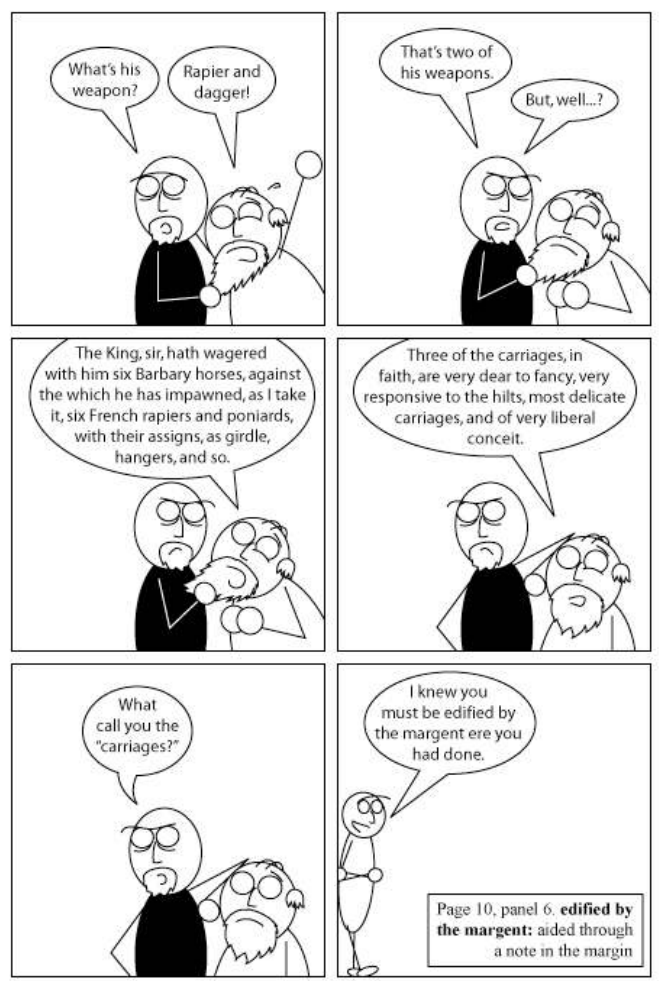

Illustration 10 : Stick Figure Hamlet, Acte V, scène ii.

Dans la même lignée, on peut mentionner l'effet de pause que Carroll insère après la première tirade un peu longue et pleine de formulations métaphoriques de la pièce (" Who is't that can inform me? / That can I », I.i.90-91), suggérant que Barnardo est luimême surpris de ce qu'il vient de dire à Horatio. C'est une façon d'anticiper la difficulté du lecteur et de le préparer aux tirades et monologues encore plus longs qui vont suivre.

D'autres ajouts puisent dans le fonds des situations codifiées propres à la bande dessinée ou au dessin animé. Ainsi, les protestations de Polonius de n'utiliser aucun art à l'acte II scène ii sont visuellement traduites par un gag rendu populaire par Tex Avery dans ses dessins animés, référence qui reparaît dans l'interview de l'auteur à travers le terme « cartoony » (Illustration 11). À la fin de la première apparition du spectre acte I, scène i, Carroll ajoute une mini-réapparition à peine perceptible en haut à gauche de l'image dans 
la lumière de l'aube naissante, qui rappelle un autre gag du même répertoire (Illustration

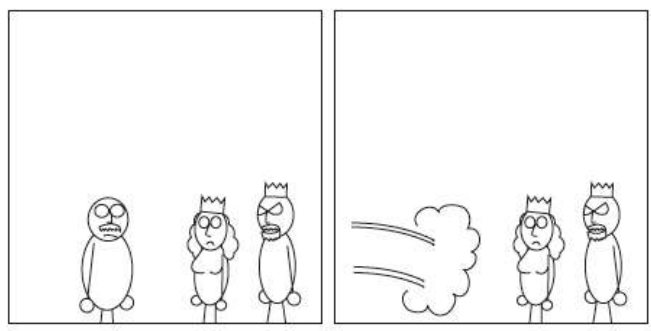

12).

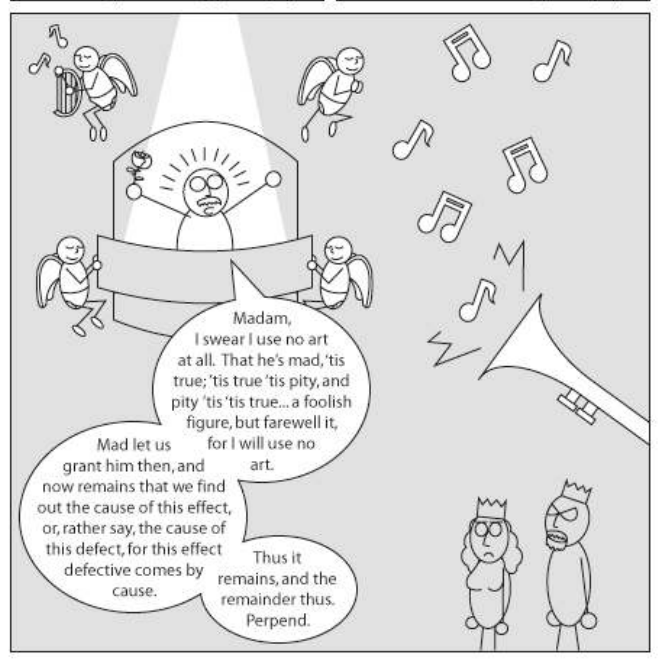

Illustration 11 : Stick Figure Hamlet, Acte II, scène ii.
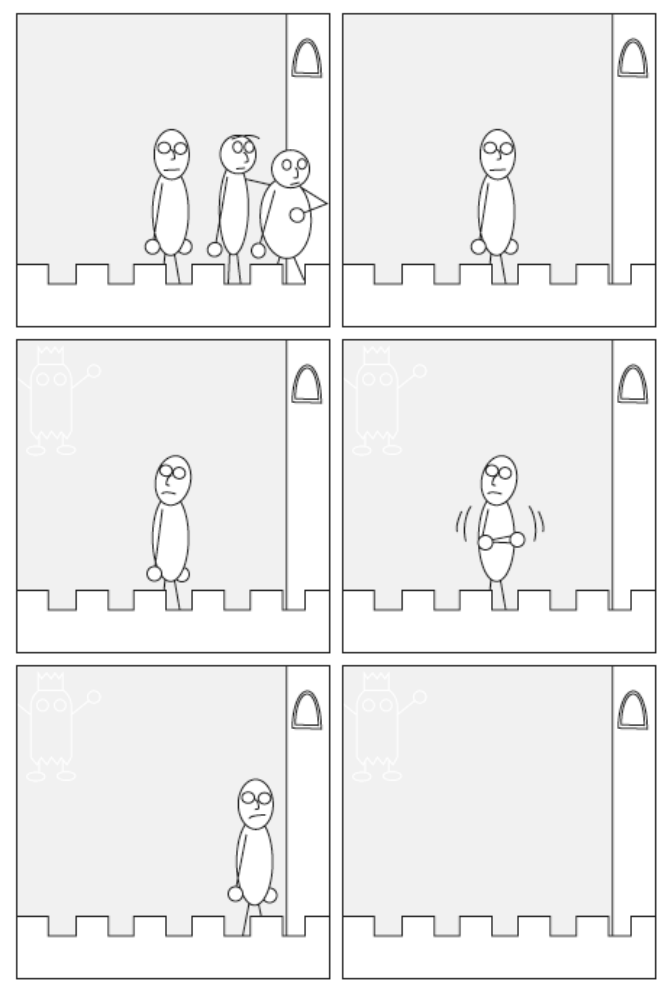
Illustration 12 : Stick Figure Hamlet, Acte I, scène i. graphique, lorsque l'image cesse d'être en adéquation avec le texte pour proposer une polyphonie qui génère non seulement du comique, mais parfois une prise de position critique par rapport au texte.

Avant que Hamlet n'aille rejoindre le spectre de son père à l'acte I, scène v, les tentatives des gardes de l'en empêcher sont transformées en bagarre agrémentée des nuages remplis d'éclairs et de signes symbolisant les coups, ainsi que de bulles rédigées en majuscules pour représenter les hurlements, deux conventions typiques de la bande dessinée (Illustration 13). Ce contrepoint entre en résonnance avec la scène de «comic relief » que Shakespeare aménage au moment du triple serment et cette résonnance s'étend également à la fin de l'entretien entre Hamlet et le spectre, lorsque Hamlet inscrit son devoir de mémoire dans ses tablettes et que Carroll nous en montre le contenu, en donnant ainsi un autre sens au vers «So, uncle, there you are» qui renvoie ici à une caricature de Claudius trépassé (Illustration 14).

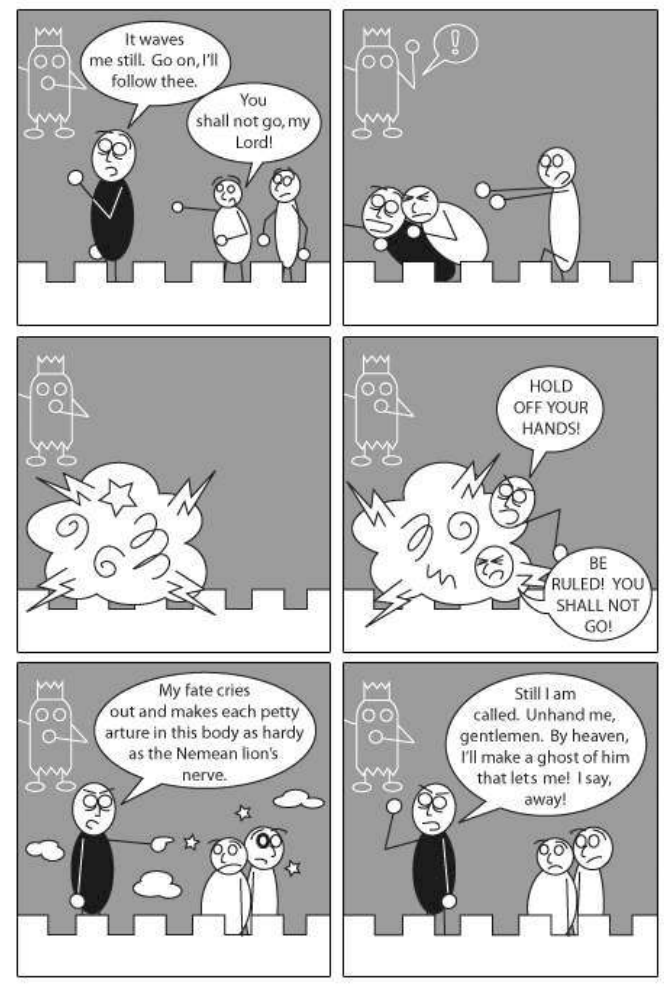


Illustration 13 : Stick Figure Hamlet, Acte I, scène iv.

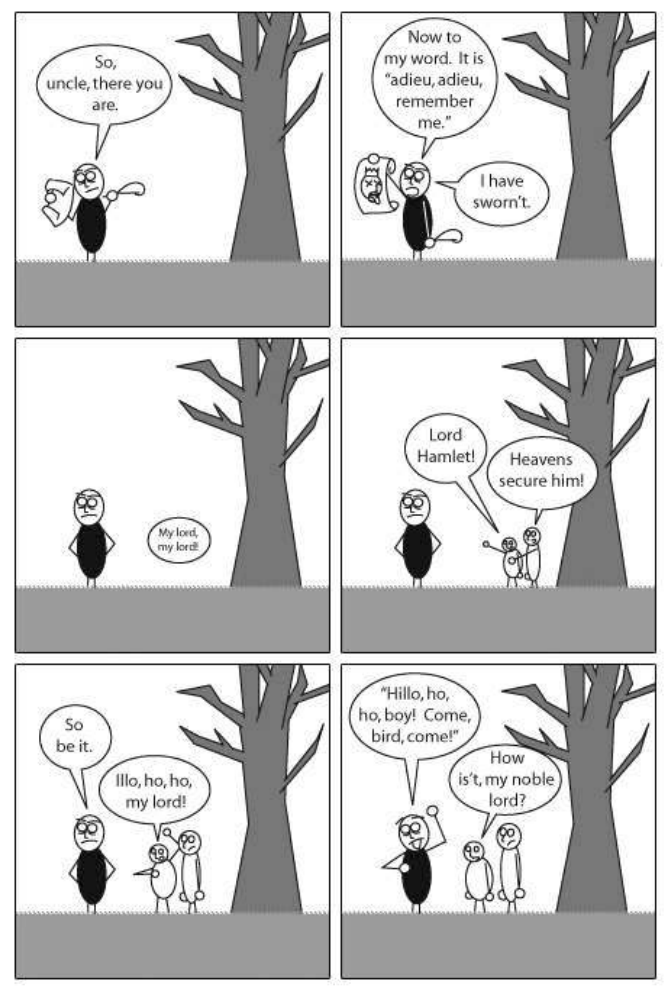

Illustration 14 : Stick Figure Hamlet, Acte I, scène v.

Il est impossible dans l'espace d'un article d'offrir un examen exhaustif de tels ornements ou détournements dont l'étendue peut varier de la simple plaisanterie, comme le marquage de l'épée empoisonnée lors du duel à l'acte $V$, scène ii avec une étiquette "not poison, honest ", à une référence plus savante, comme l'arrivée des comédiens à l'acte II, scène ii, dont le déguisement renvoie à des pièces ayant précédé Hamlet, parmi lesquelles on reconnaît A Midsummer Night's Dream et Julius Caesar (voir Illustration 9 ci-dessus), voire la combinaison d'une référence historique avec un clin d'œil à la dimension pédagogique du projet, lorsqu'à l'acte $\mathrm{V}$ scène ii, Carroll donne au roi d'Angleterre les traits d'Élisabeth $\mathrm{I}^{\mathrm{re}}$ lisant un QCM illustrant l'amitié entre le Danemark et l'Angleterre (Illustration 15). 


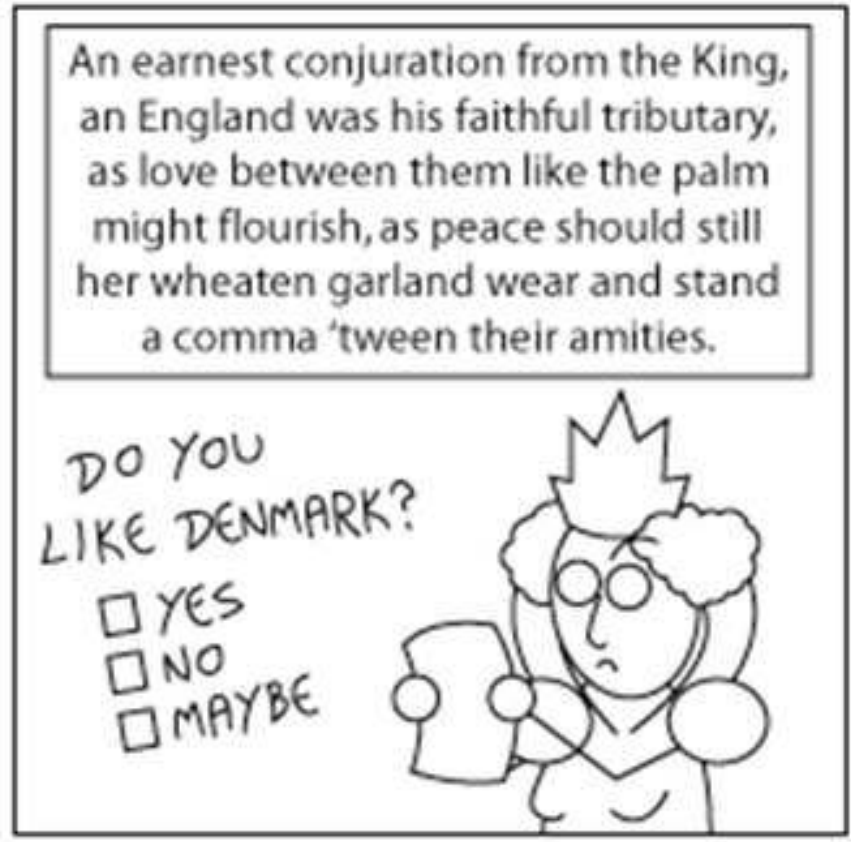

Illustration 15 : Stick Figure Hamlet, Acte V, scène ii.

Je terminerai cette analyse par un choix de mise en scène qui ne me semble tributaire d'aucune des motivations que je viens de décrire. Il concerne la pièce de résistance de toute adaptation de Hamlet, le troisième monologue du prince. Alors que Carroll choisit une façon parfaitement conventionnelle de représenter les autres monologues, tantôt laissant Hamlet seul au milieu des cases, tantôt ayant recours à la visualisation de l'hypotypose, pour "To be or not to be ", il choisit de transformer le monologue en dialogue à une voix, Hamlet s'adressant à Ophélie du début à la fin de la tirade, sous la surveillance de Polonius et Claudius, laquelle est appuyée par le changement de cadrage au milieu du monologue (Illustration 16). 

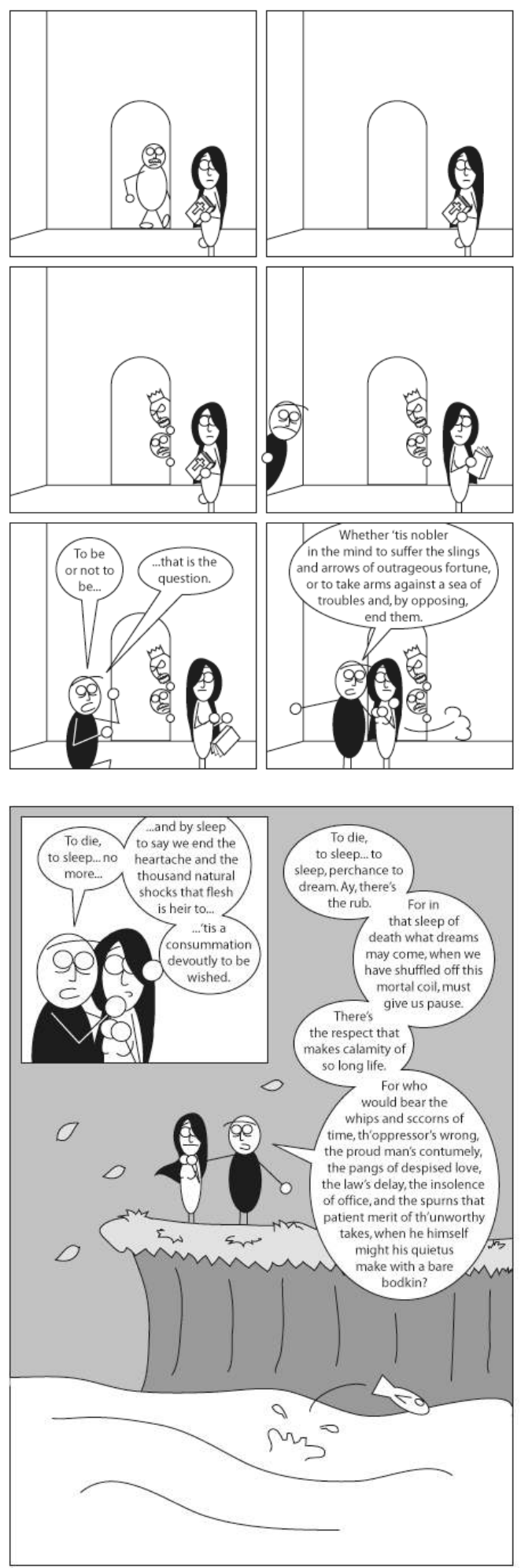


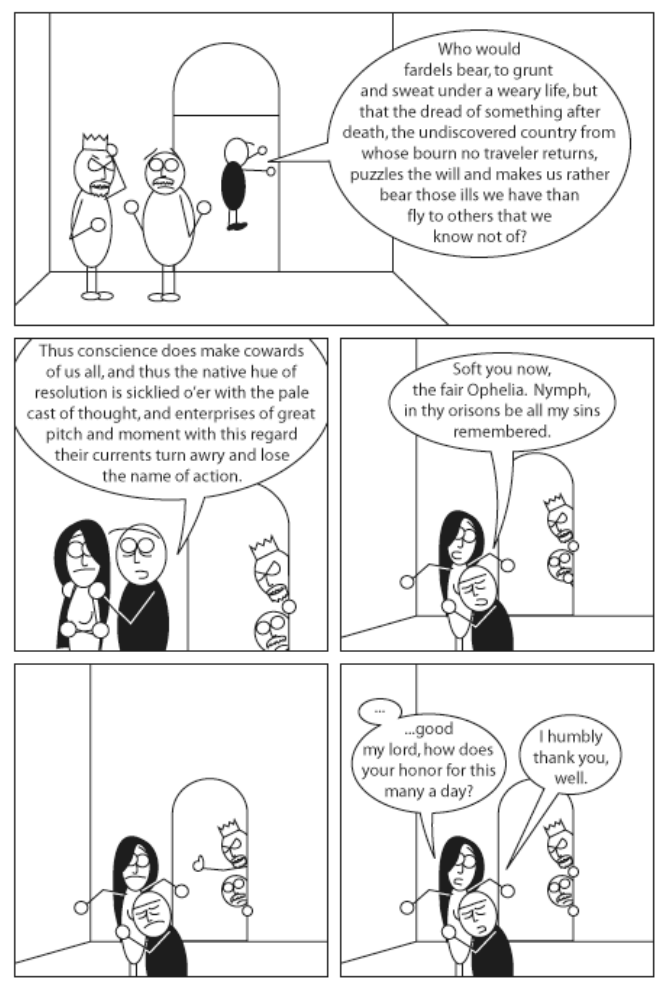

Illustration 16 : Stick Figure Hamlet, Acte III, scène i. déclenchée par l'expression de "mer de douleurs ", plaçant Hamlet et Ophélie au bord d'une falaise surplombant la mer. Mais ce qui est plus original c'est le changement de perspective qui lui succède immédiatement et qui permet à Carroll de joindre aux réactions d'Ophélie celles de Claudius et de Polonius, au moment où Hamlet évoque la crainte de l'au-delà. On ne peut s'empêcher d'établir un parallèle avec la façon dont ce monologue avait été porté à l'écran par Branagh, où, exactement au même passage du monologue, Branagh insère un contre-champ de Claudius en train d'espionner Hamlet derrière le miroir. Le fait que Carroll transforme le monologue en dialogue rend plus logique l'idée que Hamlet s'exprime à voix haute devant Ophélie et, par conséquent, que Claudius et Polonius puissent l'entendre. De plus, les deux espions affichent leur totale incompréhension de ce qu'ils entendent, renversant ainsi le rapport de pouvoir entre dominant et dominé, en phase avec les détournements ludiques de la pièce. La complexité de la mise en image du monologue « To be or not to be » montre bien que l'auteur impose ici une vision personnelle de la scène, au même titre que le ferait un metteur en scène.

De prime abord, par ses choix minimalistes, Stick Figure Hamlet pourrait paraître conforter une vision réductrice de l'œuvre de Shakespeare, mais en analysant l'ensemble de plus près, j'espère avoir montré que l'audace et la complexité de son traitement vont bien audelà d'une œuvre pour la jeunesse, à laquelle on assimile habituellement les adaptations en bande dessinée de Shakespeare. Ainsi, ce n'est pas parce qu'on s'adresse à un public plus ou moins jeune que l'adaptation de Hamlet est nécessairement superficielle. À cet égard, Stick Figure Hamlet fait preuve non seulement d'inventivité dans le détournement des clichés et icônes, mais également d'une connaissance approfondie de la pièce et témoigne de l'importance de Hamlet quel que soit le type de culture envisagé. 
En cela, Carroll est parfaitement représentatif et tributaire de l'évolution de la bande dessinée dans les dernières décennies $\mathrm{du} \mathrm{XX}^{\mathrm{e}}$ siècle, où le public recherché s'est peu à peu déplacé des enfants vers les adolescents, puis vers des jeunes adultes, glissement reflété à partir de la fin des années 1980 par la préférence des auteurs de bande dessinée pour le terme de "roman graphique ». Cette transformation du public a également renversé les rapports de la bande dessinée à l'œuvre de Shakespeare. Il n'est désormais plus uniquement question de faire découvrir des œuvres à un public qui ne peut encore les lire, mais d'offrir un regard neuf et légitime sur une œuvre connue au même titre que tout autre media.

D'ailleurs, il est intéressant d'observer chez Carroll le décalage entre la façon dont la pièce est traitée et la manière dont il en fait la publicité. Sur le site de Stick Figure Hamlet, comme sur la couverture, c'est sans surprise le crâne et la scène du cimetière qui symbolisent la pièce, mais sur le site dédié à l'ensemble du travail de Carroll ${ }^{26}$, ce même motif est détourné vers une plaisanterie: le crâne de Yorick se retrouve à l'intérieur d'une boule à neige où tantôt il sourit et tantôt fait triste mine.

Déjà en 2006, Wetmore défendait l'idée selon laquelle la bande dessinée recourt à des procédés d'adaptation et de mise en scène plus proches du théâtre que n'importe quel autre type de support, car comme le théâtre elle allie le verbe à l'image ${ }^{27}$. C'est exactement ce que l'on constate dans le travail de Dan Carroll qui, sans avoir besoin d'ajouter de commentaire textuel, se contente de traiter l'image comme le metteur en scène le fait avec le décor et la mise en scène. Et il ne semble pas être le seul. Même si, en réalité, c'est avec le cinéma (plutôt que le théâtre) que le rapprochement s'avère plus pertinent, du fait d'un point de vue unique et dirigé qu'offrent ces deux médias, c'est néanmoins le parallèle avec le théâtre que revendique une autre adaptation du texte intégral de Hamlet en bande dessinée par l'Australienne Nicki Greenberg parue en 2010, où dès la couverture, l'illustratrice entend rapprocher sa démarche de celle du metteur en scène : «William Shakespeare's Hamlet staged on the page. »

\section{NOTES}

1. Kevin J. Wetmore Jr., "'The Amazing Adventures of Superbard': Shakespeare in Comics and Graphic Novels », in Jennifer Hulbert, Kevin J. Wetmore Jr., et Robert York (éds.), Shakespeare and Youth Culture, 2006, p. 171-198. En français comme en anglais, il existe plusieurs termes pour désigner ce mode d'expression artistique classé comme le "neuvième art». En anglais, comic correspondait à l'origine à des historiettes de quelques cases qui pouvaient ensuite être réunies en volume, tandis qu'à partir des années 1970 le terme de graphic novel s'est progressivement imposé pour désigner une forme plus «sérieuse » d'ouvrages qui développaient une intrigue complexe. Ce terme fut à son tour repris en français et le "roman graphique» y a la même connotation de légitimation d'un art qui ne soit plus uniquement destiné à un public jeune. Dans le présent article, j'utilise systématiquement le terme de bande dessinée, évitant celui de roman graphique dans la mesure où celui-ci insiste sur une forme hybride (à la fois roman et œuvre graphique) ce qui a tendance à le faire paraître plus éloigné d'un texte théâtral. 
2. Toutes les références à Hamlet renvoient à l'édition en ligne de la Folger Library: http:// www.folgerdigitaltexts.org/?chapter=5\&play=Ham\&loc=p7 (consulté le 23 avril 2015).

3. Albert Uderzo et René Goscinny, La Grande traversée, Paris, Dargaud, 1975, p. 41 et 43.

4. Toutes les références renvoient au site: http://www.stickfigurehamlet.com/ (consulté le 23 avril 2015).

5. Famous Authors Illustrated: Hamlet, illustré par Henry Kiefer, adapté par Dana E. Dutch, n8, Seaboard, 1950, 36p.

6. Classics Illustrated : Hamlet, illustré par Alex A. Blum, adapté par Samuel Willinsky, First Series, n99, Gilberton, 1952, 48p.

7. Classics Illustrated: Hamlet, illustré par Tom Mandrake, adapté par Steven Grant, Second Series, n5, First Classics Inc., 1991, 56p.

8. Respectivement, Saddleback's Illustrated Classics: Hamlet, Saddleback Edu. Pub., 2003, 61p.; Picture This! Shakespeare, illustré par Christina Lacie, Hauppauge, NY, Barron's Educational Series, 2006, 80p. ; Graffex Hamlet, illustré par Penko Gele, adapté par Kathy McEvoy, Hauppauge, NY, Barrons Educational Series, 2009, 48p. ; Hamlet, illustré par Leonid Gore, adapté par Bruce Coville, New York, Dial Books, 2004, 40p.

9. Alexis et Marcel Gotlib, Hamlet, in Pilote n ${ }^{\circ} 640$ du 6 février 1972, 9p.

10. Hamlet (1976), illustré par Gianni De Luca, adapté par Barbare Graille, Les Humanoïdes associés, coll. « Noire », 1980. Parmi les innovations graphiques de De Luca, citons les planches sur lesquelles le mouvement décomposé de Hamlet reflète son état intérieur et accompagne ses soliloques. Voir Paul Gravett, « De Luca and Hamlet: Thinking Outside the Box », in European Comic $\operatorname{Art} \mathrm{n}^{\circ} 1$, Liverpool, Liverpool University Press vol. 1:1, printemps 2008, p. 20-35.

11. D'autres pièces de Shakespeare avaient déjà été adaptées dans leur intégralité, comme Othello qu'Oscar Zarate avait illustré dès 1983.

12. Respectivement, Hamlet, illustré par Emma Vieceli, New York, Amulet Books, 2007, 195p.; Hamlet, illustré par Tintin Pantoja, adapté par Adam Sexton, Indianapolis, IN, Wiley Pub., 2008, 185p.

13. No fear Shakespeare Graphic Novels: Hamlet, illustré par Neil Babra, New York, Sparknotes, 2008, 208p.

14. http://netboy.com/blog/ (consulté le 23 avril 2015).

15. Linda Hutcheon, A Theory of Adaptation, Londres, Routledge, 2006, p. 8.

16. Ibid., p. 39.

17. Pour son film, Branagh s'était servi du texte établi par G.R. Hibbard pour "The Oxford Shakespeare » et basé sur la version de l'in-folio de 1623. Je remercie Sarah Hatchuel pour cette précision.

18. Voir la documentation sur le site de Kickstarter: https://www.kickstarter.com/projects/ breadpig/to-be-or-not-to-be-that-is-the-adventure (consulté le 23 avril 2015).

19. Hutcheon, op. cit., p. 91.

20. Ibid., p. 93. Hucheon cite Marjorie Garber, Shakespeare's Ghost-Writers, Londres, Methuen, 1987, p. 7.

21. http://gapersblock.com/bookclub/2010/07/06/one-shots_dan_carroll/ (consulté le 23 avril 2015).

22. Ibid.

23. Ibid.

24. Carroll s'autorise toutefois une exception pour l'Ambassadeur qui accompagne Fortinbras dans la scène finale.

25. Carroll, op. cit.

26. http://www.herecomesyourdan.com/portal.html (consulté le 23 avril 2015).

27. "Comics have their own aesthetic and their own methods of narrative that, like theatre, combines word with image. » (Kevin J. Wetmore Jr., op. cit., p. 172). 


\section{RÉSUMÉS}

Partant d'une charte graphique minimaliste, Stick Figure Hamlet de Dan Carroll entreprend d'illustrer le texte "intégral» de Hamlet. Pour en rendre la lecture plus attractive, l'auteur introduit de nombreux « décalages » graphiques. Tantôt ceux-ci prennent la forme de références et allusions contemporaines qui établissent un contraste avec le texte classique d'Hamlet et tantôt ils offrent des situations graphiques qui, en prenant le contre-pied du texte, ajoutent une dimension humoristique à la tragédie. Ils peuvent être vus à la fois comme des choix de mise en scène (ou mise en image) et comme des stratégies et astuces dont le but est d'accompagner le lecteur dans des passages plus narratifs. Stick Figure Hamlet se distingue des autres adaptations graphiques de Hamlet conçues sur un mode plus « sérieux ", car Carroll cultive une ambivalence par rapport à son modèle : la tension entre fidélité absolue au texte original et détournements visuels fréquents constitue le cœur même de cette adaptation.

Using a minimalistic graphic style, Dan Carroll's Stick Figure Hamlet sets about illustrating the unabridged text of Hamlet. To make its reading more attractive, the author introduces graphic discrepancies. Sometimes those take the form of contemporary references or allusions, producing an anachronistic contrast with Hamlet's text and sometimes they offer graphic situations which wrong-foot the text, adding a comic dimension to the tragedy. They can be seen both as staging (or layout) choices and as strategies and devices meant to accompany the reader in longer narrative passages of the play. Stick Figure Hamlet stands out against other graphic adaptations of Hamlet created in a "serious" mode, because Carroll cultivates ambivalence towards his model: the tension between total fidelity to the original text and regular visual wrong-footing is at the very heart of this adaptation.

\section{INDEX}

Mots-clés : adaptation, bande dessinée, webcomic

Keywords : graphic novel, Hamlet

\section{AUTEUR \\ PIERRE KAPITANIAK}

Université Paris 8 